\title{
Correlação entre caracteres e estimação de parâmetros populacionais para batata-doce
}

\author{
Álvaro C Gonçalves Neto; Wilson Roberto Maluf; Luiz Antonio A Gomes; Gabriel M Maciel; Raphael de \\ Paula D Ferreira; Regis de C Carvalho \\ UFLA, Depto. Agricultura, C. Postal 3037, 37200-000 Lavras-MG; alvarocgneto@gmail.com; wrmaluf@dag.ufla.br; laagomes@dag. \\ ufla.br; gmmufla@hotmail.com; raphaelufla@hotmail.com; regisccarvalho@hotmail.com
}

\section{RESUMO}

Cultivares de batata-doce, plantadas atualmente, foram selecionadas primariamente para o consumo humano, sem levar em consideração o enorme potencial dessa cultura na produção de etanol e alimentação animal. Os objetivos deste trabalho foram estimar parâmetros populacionais e as correlações genotípicas, fenotípicas e de ambiente entre caracteres de interesse para as principais utilizações da batata-doce. Foram avaliados em blocos casualizados com duas repetições 39 genótipos de batata-doce pertencentes à coleção de germoplasma da Universidade Federal de Lavras. Os coeficientes de variação genética $\left(\mathrm{CV}_{\mathrm{g}}\right)$ e ambiental $\left(\mathrm{CV}_{\mathrm{e}}\right)$, herdabilidades no sentido amplo $\left(\mathrm{h}_{\mathrm{a}}{ }^{2}\right)$ e a razão $\mathrm{b}=\mathrm{CV}_{\mathrm{g}} / \mathrm{CV}_{\mathrm{e}}$ indicam uma situação favorável para a seleção da maioria das características analisadas, sendo os valores no geral superiores a 9,52\%, 1,44 e 79,31\% para $\mathrm{CV}_{\mathrm{g}}, \mathrm{CV}_{\mathrm{g}} /$ $\mathrm{CV}_{\mathrm{e}} \mathrm{e} \mathrm{h}_{\mathrm{a}}^{2}$, respectivamente. Em todos os pares de caracteres estudados os coeficientes de correlação genotípica e fenotípica, além de ser de mesmo sinal, foram semelhantes na magnitude e no nível de significância. Nas estimativas que apresentaram correlações significativas, as correlações genotípicas foram ligeiramente superiores às fenotípicas, e ambas foram superiores às correlações de ambiente, como em produção total de raízes frescas x porcentagem de massa seca das raízes; porcentagem de massa seca na parte aérea $\mathrm{x}$ produção total de raízes frescas; nota para formato médio de raízes x nota para formato geral de raízes. Não se verificou correlação genética ou fenotípica entre porcentagem de massa seca das raízes e densidade de raízes. A seleção e recomendação de genótipos de batata-doce baseada no conjunto dessas características estudadas (dependendo do segmento de mercado a ser explorado) possibilitará a plena utilização deste valioso recurso genético vegetal, e a sua adoção mais intensa na agricultura nacional.

Palavras-chave: Ipomoea batatas, alimentação animal, produção de etanol, herdabilidade.

\section{ABSTRACT \\ Correlation between traits and estimate of population parameters for sweetpotato}

Sweetpotato cultivars currently grown were selected essentially for their use in human consumption, with little regard for their other potential uses such as ethanol production or animal feed. The objective of this work was to estimate population parameters and genotypic, phenotypic and environmental correlations associated with these traits. Thirty-nine sweetpotato genotypes from the germplasm bank of the Universidade Federal de Lavras were assayed in a randomized complete block design trial with 2 replications. The estimated genetic $\left(\mathrm{CV}_{\mathrm{g}}\right)$ and environmental $\left(\mathrm{CV}_{\mathrm{e}}\right)$ coefficients of variation, the broad sense heritabilities $\left(\mathrm{h}_{\mathrm{a}}^{2}\right)$ and the $\mathrm{b}=\mathrm{CV}_{\mathrm{g}} / \mathrm{CV}_{\mathrm{e}}$ ratio favor selection of the majority of the traits analysed, with estimates generally higher than $9.52 \%, 1.44$ and $79.31 \%$ for $\mathrm{CV}_{\mathrm{g}}, \mathrm{CV}_{\mathrm{g}} / \mathrm{CV}_{\mathrm{e}}$ and $\mathrm{h}_{\mathrm{a}}{ }^{2}$, respectively. For all pairs of traits studied, the genotypic and phenotypic correlations had the same sign, and were similar in both magnitude and level of significance. In the cases where correlations were significant, the estimates of the genotypic correlations were slightly higher than those of the phenotypic correlations, and both were higher than environmental correlations as was observed between total fresh root yield $\mathrm{x}$ percentage of dry matter in roots, percentage of dry matter in the leaves+vines $\mathrm{x}$ total fresh root yield, mean root shape ratings $x$ general root shape ratings. No genetic or phenotypic correlation was observed beetween percentage of root dry matter and measured root density. The selection and recommendation of sweet potato genotypes based on a set of these studied characteristics (depending on the segment of market to be explored), will make possible the complete use of this precious vegetable genetic resource, and its more intense adoption in national agriculture.

Keywords: Ipomoea batatas, animal food, ethanol production, heritabilities.

(Recebido para publicação em 6 de setembro de 2011; aceito em 16 de outubro de 2012) (Received on September 6, 2011; accepted on October 16, 2012)

\begin{abstract}
$\mathrm{A}$ batata-doce (Ipomoea batatas), apesar de seu alto potencial como produtora de alimentos e de biomassa (superior a $40 \mathrm{t} \mathrm{ha}^{-1}$ de raízes em ciclo de 6 meses) (Silveira et al., 2007), no Brasil, ainda se caracteriza pela baixa produtividade (cerca de $12 \mathrm{tha}^{-1}$ ) (IBGE, 2010). Por apresentar grande variabilidade genética, a batata-doce permite seleção para inúmeros propósitos (Oliveira et al., 2000; Gonçalves Neto et al.,
\end{abstract}

2011; Neiva et al., 2011), podendo ser destinada tanto à alimentação humana, como também para alimentação animal e produção de etanol.

No Brasil, a utilização de ramas de batata-doce na alimentação animal é feita em escala bastante limitada. Presume-se que a maior parte das ramas é simplesmente descartada como resíduo inaproveitável, exceto material propagativo que é utilizado para implantação de novos plantios.

Ainda pouco utilizada para esta finalidade, a batata-doce talvez seja uma das espécies vegetais que podem apresentar os melhores resultados para a produção de etanol no Brasil. A cana-de-açúcar, com elevada produtividade (100 t ha ${ }^{-1}$ de colmos e $90 \mathrm{~L}$ de etanol por tonelada), atinge a produção de $9.000 \mathrm{~L} \mathrm{ha}^{-1}$ de etanol, em ciclo de 12 meses (Bioetanol de cana de açúcar, 2008). Gonçalves Neto 
et al., (2011), em genótipos de batata-doce considerados aptos para produção de etanol, com base na produção de 158 L de etanol por tonelada de raízes processadas relatado por Silveira (2008), obtiveram produção etanólica de até 15.484,0 $\mathrm{L} \mathrm{ha}^{-1}$ em ciclo de sete meses. Sabendo que todo parque tecnológico é voltado para produção de etanol a partir da cana-de-acúçar, fica claro neste momento a viabilidade do uso da batata-doce para produção de biocombustível de segunda geração, sem a intenção de substituir a cana, mas complementar a produção de etanol. Pois, a batata-doce pode produzir álcool com melhores propriedades químicas, produto de alto valor agregado, destinado à fabricação de cosmético, tintas e remédios (Castro et al., 2008).

São evidentes os múltiplos usos da batata-doce, entretanto, a seleção para apenas um desses usos pode levar à inaptidão agronômica para outras finalidades, pois a seleção com base em um ou poucos caracteres pode resultar em alterações desfavoráveis nos demais, já que existem correlações genéticas negativas entre eles. Quase todas as cultivares de batata-doce, plantadas atualmente, foram selecionadas apenas para o consumo humano, sem levar em consideração o enorme potencial desta cultura para produção de etanol e alimentação animal, tornando necessário, a identificação de genótipos que apresentem conjuntos de caracteres de interesse para cada uma das aptidões (Gonçalves Neto et al., 2011).

Para explorar todo seu potencial produtivo, vários são os caracteres de interesse na cultura da batata-doce, portanto, indispensável é a investigação das correlações genéticas, fenotípicas e ambientais existentes entre estes caracteres. A seleção indireta por meio de caracteres correlacionados permite que caracteres mais complexos ou de difícil mensuração, sejam selecionados por meio da seleção no caráter associado. Isso é vantajoso, principalmente quando um caráter com alto valor agrícola é de baixa herdabilidade (Ferreira et al., 2003). No caso da batata-doce, a herdabilidade no sentido amplo é importante devido aos efeitos de dominância e epistasia serem mantidos pela propagação vegetativa.

Dessa forma, este trabalho teve como objetivos estimar parâmetros populacionais e as correlações genotípicas, fenotípicas e de ambiente entre caracteres de interesse para as principais utilizações da batata-doce (consumo humano, alimentação animal e produção de etanol), de modo a orientar programas de melhoramento genético desta cultura.

\section{MATERIAL E MÉTODOS}

Um total de 39 genótipos de batata-doce foram avaliados, sendo 36 clones e três cultivares comerciais pertencentes à coleção de germoplasma da UFLA (Tabela 1).

O experimento foi instalado no campo da empresa HortiAgro Sementes Ltda., localizada na Fazenda Palmital,

Tabela1. Descrição dos clones de batata-doce, utilizados no experimento (description of the sweetpotato clones tested in the trial). Lavras, UFLA, 2007.

\begin{tabular}{|c|c|c|c|c|c|}
\hline Código & Nome & Procedência & Código & Nome & Procedência \\
\hline & Palmas* & $\mathrm{UFT}^{-\mathrm{TO}^{\prime 3}}$ & UFLA07-21 & DIA-BD-65 & UFVJM-MG \\
\hline & Brazlândia-Branca* & EMBRAPA & UFLA07-22 & DIA-BD-46 & UFVJM-MG \\
\hline & Brazlândia-Rosada* & EMBRAPA & UFLA07-23 & UFT-BD-14-Al & UFT-TO $^{\prime 3}$ \\
\hline UFLA07-01 & Coracão-Magoado & UFVJM-MG'/2 & UFLA07-24 & DIA-BD-67 & UFVJM-MG \\
\hline UFLA07-02 & DIA-BD-07 & UFVJM-MG & UFLA07-26 & DIA-BD-31 & UFVJM-MG \\
\hline UFLA07-03 & Cambraia & UFVJM-MG & UFLA07-27 & DIA-BD-14 & UFVJM-MG \\
\hline UFLA07-04 & Arruba & UFVJM-MG & UFLA07-29 & DIA-BD-11 & UFVJM-MG \\
\hline UFLA07-05 & Licuri & UFVJM-MG & UFLA07-31 & DIA-BD-38 & UFVJM-MG \\
\hline UFLA07-08 & DIA-BD-24 & UFVJM-MG & UFLA07-37 & UFT-BD-115 & UFT-TO \\
\hline UFLA07-09 & Princesa & UFVJM-MG & UFLA07-40 & UFT-BD-35-Al & UFT-TO \\
\hline UFLA07-10 & DIA-BD-12 & UFVJM-MG & UFLA07-41 & DIA-BD-54 & UFVJM-MG \\
\hline UFLA07-11 & Tomba-carro & UFVJM-MG & UFLA07-42 & UFT-BD-58 & UFT-TO \\
\hline UFLA07-12 & DIA-BD-06 & UFVJM-MG & UFLA07-43 & UFT-BD-02-Al & UFT-TO \\
\hline UFLA07-14 & DIA-BD-56 & UFVJM-MG & UFLA07-45 & UFT-BD-09-Al & UFT-TO \\
\hline UFLA07-15 & DIA-BD-42 & UFVJM-MG & UFLA07-46 & UFT-BD-52 & UFT-TO \\
\hline UFLA07-16 & DIA-BD-15 & UFVJM-MG & UFLA07-48 & Canuanã & UFT-TO \\
\hline UFLA07-17 & DIA-BD-25 & UFVJM-MG & UFLA07-49 & UFT-BD-04-Al & UFT-TO \\
\hline UFLA07-18 & Marmel & UFVJM-MG & UFLA07-51 & UFT-BD-100 & UFT-TO \\
\hline UFLA07-19 & DIA-BD-45 & UFVJM-MG & UFLA07-53 & UFT-BD-08 & UFT-TO \\
\hline UFLA07-20 & DIA-BD-39 & UFVJM-MG & - & - & - \\
\hline
\end{tabular}

${ }^{*}$ Cultivares comerciais (testemunhas) (commercial cultivars (control treatments)); ${ }^{1}$ Embrapa Hortaliças (CNPH); ${ }^{2}$ Universidade Federal dos Vales do Jequitinhonha e Mucurí (UFVJM); ${ }^{3}$ Universidade Federal do Tocantins (UFT). 
no município de Ijaci-MG $\left(21^{\circ} 14^{\prime} 16^{\prime \prime} \mathrm{S}\right.$, 4508'00"O, altitude de $918 \mathrm{~m}$ ), de março a outubro de 2007. O solo, que apresentava acidez corrigida e média fertilidade, foi submetido a uma aração e a duas gradagens. Em seguida, foram levantados camalhões com $40 \mathrm{~cm}$ de altura, adubados com $1.000 \mathrm{~kg} \mathrm{ha}^{-1} \mathrm{da}$ fórmula N-P-K (4-14-8). Dois meses após o plantio, foi realizada adubação de cobertura com $1.000 \mathrm{~kg} \mathrm{ha}^{-1}$ de ureia, seguindo recomendação da análise de solo. O delineamento experimental utilizado foi em blocos casualizados com duas repetições. As parcelas foram compostas de leiras com 12 plantas, no espaçamento de $0,80 \mathrm{~m}$ entre leiras por $0,40 \mathrm{~m}$ entre plantas (cada parcela com total de 3,84 $\mathrm{m}^{2}$ de área útil), com utilização de bordadura nas laterais do experimento. No plantio, utilizaram-se partes do caule da batata-doce com aproximadamente seis gemas, das quais três foram enterradas no solo. O experimento foi conduzido sob condições de irrigação por aspersão e a colheita realizada sete meses após o plantio. Foram avaliados os seguintes caracteres:

Produção total de raízes frescas - massa total de raízes colhidas por parcela (em seguida transformados para $\left.\mathrm{t} \mathrm{ha}^{-1}\right)$; Massa seca nas raízes (\%) - amostras com cerca de 1,5 kg de raízes de cada parcela foram trituradas e secas em estufa a $65^{\circ} \mathrm{C}$, até atingir massa constante $=($ massa seca $/$ massa fresca $) \mathrm{x}$ 100; Produção de massa seca nas raízes - obtida pela relação entre produção total de raízes frescas e porcentagem de massa seca nas raízes $\left(\mathrm{t} \mathrm{ha}^{-1}\right)$; Densidade das raízes - determinada pelo método descrito pelo Centro Internacional de La Papa (2001): as raízes tuberosas de cada clone foram lavadas para retirar o excesso de solo, secas com papel toalha e, em seguida procedeu-se à pesagem de cerca de $2 \mathrm{~kg}$ de raízes no ar; posteriormente foi feita a pesagem na água, em balança hidrostática, determinando-se assim a densidade com base na seguinte fórmula: Densidade $=$ massa no ar/(massa no ar - massa na água); Produção de massa fresca de parte aérea - a parte aérea de cada parcela foi cortada ao nível do solo na leira e pesada para obtenção da massa fresca total (ramas+folhas) $\left(\mathrm{t} \mathrm{ha} \mathrm{h}^{-1}\right)$; Massa seca na parte aérea
(\%) - amostras de aproximadamente 1,5 $\mathrm{kg}$ de parte aérea foram trituradas, em seguida foram secas em estufa a $65^{\circ} \mathrm{C}$, até atingir massa constante para determinação da massa seca $(\%)=($ massa seca/massa fresca) x 100; Produção de massa seca na parte aérea - a partir dos dados obtidos (produção de massa fresca de parte aérea e porcentagem de massa seca na parte aérea) foi calculada a produção total de massa seca na parte aérea $\left(\mathrm{t} \mathrm{ha} \mathrm{h}^{-1}\right)$; Produção de raízes comerciais - foram consideradas comerciais aquelas de formato uniforme, lisas com massa igual ou superior a $100 \mathrm{~g}$, e não-comerciais as raízes muito longas (mais de $30 \mathrm{~cm}$ ), tortuosas, com veias, muito danificadas por insetos ou por cortes e esfolamentos (Miranda et al., 1995); Formato médio de raízes - foi determinado por meio de uma escala de notas que variou de 1 (excelente) a 5 (péssimo), citado por Azevedo et al. (2000). Foram avaliadas entre $4 \mathrm{e}$ 10 raízes individualmente, tomando-se como nota da parcela a média de todas as raízes avaliadas na respectiva parcela; Formato geral de raízes - determinado por meio de uma escala de notas que variou de 1 (excelente) a 5 (péssimo), citado por Azevedo et al. (2000). Apenas uma nota geral foi atribuída à parcela considerando todas as raízes das respectivas parcelas; Teor de sólidos solúveis nas raízes - raízes das batatas-doce foram trituradas em moinho de carne, em seguida os pedaços foram espremidos usando-se um espremedor manual de limão, o suco obtido foi colocado em um refratômetro digital (ATAGO) para que se procedesse à leitura do teor de sólidos solúveis, expresso em graus Brix.

Os dados foram submetidos à análise de variância para cada caráter e, em seguida, as médias foram agrupadas por meio do teste de Scott-Knott, a 5\% de probabilidade. A partir dos componentes de variância foram estimados a herdabilidade no sentido amplo $\left(\mathrm{h}_{\mathrm{a}}^{2}\right)$ e os coeficientes de correlação fenotípica $\left(r_{F}\right)$, genotípica $\left(r_{G}\right)$ e ambiental $\left(r_{A}\right)$ referentes às características mais relevantes. Em seguida foi empregado o método de bootstrap (Efron \& Tibshirani, 1993) com 10.000 simulações para verificar a significância estatística das estimativas das correlações ao nível de 1 e 5\% de probabilidade. Para as correlações fenotípicas também foi empregado o teste $t$. As análises estatísticas foram realizadas utilizando o pacote computacional SAS Institute (2001).

\section{RESULTADOS E DISCUSSÃO}

Na produção total de raízes frescas, destacaram-se os clones UFLA07-04 $\left(52,70 \mathrm{t} \mathrm{ha}^{-1}\right)$, UFLA07-31 (56,80 $\left.\mathrm{tha}^{-1}\right)$, UFLA07-49 (64,30 $\left.\mathrm{t} \mathrm{ha}^{-1}\right)$, e, principalmente, os clones UFLA07-12 (97,99 t ha $\left.^{-1}\right)$, UFLA07-43 $\left(95,09 \mathrm{tha}^{-1}\right)$, de duas a três vezes mais produtivos que as três testemunhas Palmas, Brazlândia-Branca e Brazlandia-Rosada, que produziram, respectivamente, 27,30; 28,00 e 11,30 t ha $^{-1}$ (Tabela 2). Os valores máximos de produtividade obtidos são superiores aos encontrados por outros autores (Azevedo et al., 2000; Cardoso et al., 2005; Oliveira et al., 2006; Silveira, 2008), em que a produtividade máxima atingida foi de $65,5 \mathrm{t} \mathrm{ha}^{-1}$, com colheita aos 6 meses.

A porcentagem de massa seca nas raízes variou entre 21,47 e $45,10 \%$. Clones como UFLA07-21, UFLA07-22, UFLA07-26 e UFLA07-41 ultrapassaram a marca de $40 \%$ de massa seca (Tabela 2). Elevadas porcentagens de massa seca tendem a ser proporcionais à porcentagem de amido nas raízes, característica diretamente relacionada com o rendimento em etanol por tonelada de raiz processada (Silveira, 2008).

Na produção de massa seca nas raízes, os melhores clones foram UFLA0749, UFLA07-12 e UFLA07-43, que produziram, respectivamente 21,$00 ; 23,20$ e 31,60 t ha-1 de massa seca nas raízes.

Os clones UFLA07-21 e UFLA0745 apresentaram maiores densidades de raízes, diferindo estatisticamente dos demais (Tabela 2). Os valores variaram entre 1,0231 e 1,0993, resultados próximos aos encontrados por Cardoso et al. (2007), que testando clones de batatas doce encontraram densidade de raízes entre 1,00 e 1,08. Existe relação matemática entre densidade e porcentagem mássica de água para algumas amiláceas como, Solanum tuberosum (batata-inglesa), Dioscorea sp (cará), Manihot utilíssima (mandioca), quando frescos 
Tabela 2. Médias das características, produção de raízes frescas, massa seca das raízes (\%), produção de massa seca nas raízes, densidade de raízes, massa fresca de parte aérea, massa seca na parte aérea (\%), produção de massa seca na parte aérea, produção de raízes comerciais, formato de raízes, em clones de batata-doce (average values of total fresh matter of roots, dry matter of roots (\%), dry matter of roots, root densities, fresh matter of vine+leaves, dry matter of vines+leaves (\%), total dry matter of vines+leaves, root shape of sweetpotato clones). Lavras, UFLA, 2007.

\begin{tabular}{|c|c|c|c|c|c|c|c|c|c|}
\hline Código & $\begin{array}{l}\text { Raízes } \\
\text { frescas } \\
\left(t \text { ha }^{-1}\right)\end{array}$ & $\begin{array}{c}\text { Massa seca } \\
\text { de raízes } \\
(\%)\end{array}$ & $\begin{array}{c}\text { Massa seca } \\
\text { de raízes } \\
\left(\mathrm{t} \mathrm{ha}^{-1}\right)\end{array}$ & $\begin{array}{l}\text { Densidade } \\
\text { raízes }\end{array}$ & $\begin{array}{c}\text { Massa fresca } \\
\text { (parte aérea) } \\
\left(\mathrm{t} \mathrm{ha}^{-1}\right)\end{array}$ & $\begin{array}{c}\text { Massa seca } \\
\text { (p. aérea) } \\
\text { (\%) }\end{array}$ & $\begin{array}{c}\text { Massa seca } \\
\text { (p. aérea) } \\
\left(\mathrm{t} \mathrm{ha}^{-1}\right)\end{array}$ & $\begin{array}{c}\text { Raízes co- } \\
\text { merciais } \\
\left(\mathrm{t} \mathrm{ha}^{-1}\right)\end{array}$ & $\begin{array}{c}\text { Raízes } \\
\text { Formato }^{2}\end{array}$ \\
\hline Palmas $^{1}$ & $27,30 \mathrm{~d}$ & $33,75 \mathrm{~b}$ & $9,15 \mathrm{~d}$ & $1,0565 \mathrm{~b}$ & $55,10 \mathrm{e}$ & $15,67 \mathrm{a}$ & $8,61 \mathrm{c}$ & $22,85 \mathrm{~b}$ & $3,5 \mathrm{~b}$ \\
\hline $\begin{array}{l}\text { Brazlandia- } \\
\text {-Branca }^{1}\end{array}$ & $28,00 \mathrm{~d}$ & $30,35 \mathrm{c}$ & $8,50 \mathrm{~d}$ & $1,0524 \mathrm{c}$ & $39,60 \mathrm{e}$ & $14,12 \mathrm{a}$ & $5,50 \mathrm{~d}$ & $24,60 \mathrm{~b}$ & $3,0 \mathrm{a}$ \\
\hline $\begin{array}{l}\text { Brazlandia- } \\
\text {-Rosada }{ }^{1}\end{array}$ & $11,30 \mathrm{e}$ & $37,45 \mathrm{~b}$ & $4,25 \mathrm{e}$ & $1,0411 \mathrm{c}$ & $101,80 \mathrm{~d}$ & $19,63 \mathrm{a}$ & $20,01 \mathrm{~b}$ & $7,80 \mathrm{c}$ & $3,0 \mathrm{a}$ \\
\hline UFLA07-01 & $42,40 \mathrm{c}$ & $28,05 \mathrm{c}$ & $12,05 \mathrm{c}$ & $1,0231 \mathrm{~d}$ & $39,15 \mathrm{e}$ & $16,69 \mathrm{a}$ & $6,66 \mathrm{~d}$ & $30,40 \mathrm{~b}$ & $3,5 \mathrm{~b}$ \\
\hline UFLA07-02 & $12,80 \mathrm{e}$ & $38,60 \mathrm{a}$ & $4,95 \mathrm{e}$ & $1,0472 \mathrm{c}$ & $40,00 \mathrm{e}$ & $15,35 \mathrm{a}$ & $6,21 \mathrm{~d}$ & $9,05 \mathrm{c}$ & $2,5 \mathrm{a}$ \\
\hline UFLA07-03 & $24,70 \mathrm{~d}$ & $32,50 \mathrm{~b}$ & $7,85 \mathrm{~d}$ & $1,0540 \mathrm{~b}$ & $13,95 \mathrm{e}$ & $19,53 \mathrm{a}$ & $2,74 \mathrm{~d}$ & $8,50 \mathrm{c}$ & $3,5 \mathrm{~b}$ \\
\hline UFLA07-04 & $52,70 \mathrm{~b}$ & $24,77 \mathrm{~d}$ & $12,86 \mathrm{c}$ & $1,0302 \mathrm{~d}$ & $76,84 \mathrm{~d}$ & $13,93 \mathrm{a}$ & $10,30 \mathrm{c}$ & $16,53 \mathrm{c}$ & $2,9 \mathrm{a}$ \\
\hline UFLA07-05 & $44,80 \mathrm{c}$ & $31,90 \mathrm{c}$ & $14,30 \mathrm{c}$ & $1,0429 \mathrm{c}$ & $76,25 \mathrm{~d}$ & $18,23 \mathrm{a}$ & $14,21 \mathrm{c}$ & $26,75 \mathrm{~b}$ & $3,5 \mathrm{~b}$ \\
\hline UFLA07-08 & $17,20 \mathrm{~d}$ & $30,95 \mathrm{c}$ & $5,35 \mathrm{e}$ & $1,0437 \mathrm{c}$ & $201,55 \mathrm{~b}$ & $17,18 \mathrm{a}$ & 34,83 a & $7,45 \mathrm{c}$ & $2,5 \mathrm{a}$ \\
\hline UFLA07-09 & $17,10 \mathrm{~d}$ & $24,07 \mathrm{~d}$ & $3,96 \mathrm{e}$ & $1,0605 \mathrm{~b}$ & $33,84 \mathrm{e}$ & $14,75 \mathrm{a}$ & $4,25 \mathrm{~d}$ & $12,13 \mathrm{c}$ & $1,9 \mathrm{a}$ \\
\hline UFLA07-10 & $46,70 \mathrm{c}$ & $31,35 \mathrm{c}$ & $14,65 \mathrm{c}$ & $1,0717 \mathrm{~b}$ & $73,00 \mathrm{~d}$ & $14,91 \mathrm{a}$ & $10,71 \mathrm{c}$ & $19,30 \mathrm{~b}$ & $5,0 \mathrm{c}$ \\
\hline UFLA07-11 & $17,40 \mathrm{~d}$ & $32,60 \mathrm{~b}$ & $5,70 \mathrm{~d}$ & $1,0336 \mathrm{~d}$ & $102,85 \mathrm{~d}$ & $16,28 \mathrm{a}$ & $16,59 \mathrm{~b}$ & $6,75 \mathrm{c}$ & $2,5 \mathrm{a}$ \\
\hline UFLA07-12 & $98,00 \mathrm{a}$ & $23,60 \mathrm{~d}$ & $23,20 \mathrm{~b}$ & $1,0369 \mathrm{~d}$ & $136,85 \mathrm{c}$ & $14,97 \mathrm{a}$ & $20,00 \mathrm{~b}$ & $51,35 \mathrm{a}$ & $2,0 \mathrm{a}$ \\
\hline UFLA07-14 & $3,70 \mathrm{e}$ & $21,47 \mathrm{~d}$ & $0,55 \mathrm{e}$ & $1,0349 \mathrm{~d}$ & $15,04 \mathrm{e}$ & $14,27 \mathrm{a}$ & $1,24 \mathrm{~d}$ & $1,03 \mathrm{c}$ & $2,9 \mathrm{a}$ \\
\hline UFLA07-15 & $30,80 \mathrm{~d}$ & $32,55 \mathrm{~b}$ & $9,95 \mathrm{~d}$ & $1,0700 \mathrm{~b}$ & $302,45 \mathrm{a}$ & $11,45 \mathrm{a}$ & $34,41 \mathrm{a}$ & $12,20 \mathrm{c}$ & $3,0 \mathrm{a}$ \\
\hline UFLA07-16 & $45,70 \mathrm{c}$ & $26,65 \mathrm{c}$ & $12,00 \mathrm{c}$ & $1,0257 \mathrm{~d}$ & $51,95 \mathrm{e}$ & $15,13 \mathrm{a}$ & $7,84 \mathrm{c}$ & $30,65 \mathrm{~b}$ & $3,0 \mathrm{a}$ \\
\hline UFLA07-17 & $6,00 \mathrm{e}$ & $33,45 \mathrm{~b}$ & $2,00 \mathrm{e}$ & $1,0493 \mathrm{c}$ & $67,00 \mathrm{~d}$ & $18,83 \mathrm{a}$ & $12,86 \mathrm{c}$ & $3,70 \mathrm{c}$ & $3,5 \mathrm{~b}$ \\
\hline UFLA07-18 & $28,40 \mathrm{~d}$ & $35,30 \mathrm{~b}$ & $10,10 \mathrm{~d}$ & $1,0323 \mathrm{~d}$ & $160,10 \mathrm{c}$ & $14,79 \mathrm{a}$ & $23,68 \mathrm{~b}$ & $8,35 \mathrm{c}$ & $5,0 \mathrm{c}$ \\
\hline UFLA07-19 & $26,90 \mathrm{~d}$ & $29,80 \mathrm{c}$ & $7,65 \mathrm{~d}$ & $1,0510 \mathrm{c}$ & $84,70 \mathrm{~d}$ & $12,63 \mathrm{a}$ & $10,61 \mathrm{c}$ & $14,50 \mathrm{c}$ & $2,0 \mathrm{a}$ \\
\hline UFLA07-20 & $8,20 \mathrm{e}$ & $34,25 \mathrm{~b}$ & $2,80 \mathrm{e}$ & $1,0553 \mathrm{~b}$ & $22,25 \mathrm{e}$ & $19,56 \mathrm{a}$ & $4,35 \mathrm{~d}$ & $4,90 \mathrm{c}$ & $3,0 \mathrm{a}$ \\
\hline UFLA07-21 & $15,50 \mathrm{~d}$ & $45,10 \mathrm{a}$ & $6,95 \mathrm{~d}$ & $1,0910 \mathrm{a}$ & $219,85 \mathrm{~b}$ & $16,15 \mathrm{a}$ & $36,08 \mathrm{a}$ & $5,10 \mathrm{c}$ & $5,0 \mathrm{c}$ \\
\hline UFLA07-22 & $6,10 \mathrm{e}$ & $43,57 \mathrm{a}$ & $2,96 \mathrm{e}$ & $1,0420 \mathrm{c}$ & $7,74 \mathrm{e}$ & $18,35 \mathrm{a}$ & $0,55 \mathrm{~d}$ & $5,63 \mathrm{c}$ & $4,9 \mathrm{c}$ \\
\hline UFLA07-23 & $8,20 \mathrm{e}$ & $34,05 \mathrm{~b}$ & $2,85 \mathrm{e}$ & $1,0361 \mathrm{~d}$ & $29,45 \mathrm{e}$ & $19,57 \mathrm{a}$ & $5,91 \mathrm{~d}$ & $6,75 \mathrm{c}$ & $3,0 \mathrm{a}$ \\
\hline UFLA07-24 & $46,20 \mathrm{c}$ & $30,85 \mathrm{c}$ & $14,30 \mathrm{c}$ & $1,0409 \mathrm{c}$ & $236,45 \mathrm{~b}$ & $15,00 \mathrm{a}$ & $36,10 \mathrm{a}$ & $23,50 \mathrm{~b}$ & $2,0 \mathrm{a}$ \\
\hline UFLA07-26 & $3,00 \mathrm{e}$ & $42,77 \mathrm{a}$ & $1,66 \mathrm{e}$ & $1,0472 \mathrm{c}$ & $20,84 \mathrm{e}$ & $14,72 \mathrm{a}$ & $2,21 \mathrm{~d}$ & $2,13 \mathrm{c}$ & $4,9 \mathrm{c}$ \\
\hline UFLA07-27 & $44,90 \mathrm{c}$ & $29,40 \mathrm{c}$ & $13,05 \mathrm{c}$ & $1,0524 \mathrm{c}$ & $146,45 \mathrm{c}$ & $18,40 \mathrm{a}$ & $26,80 \mathrm{~b}$ & $27,35 \mathrm{~b}$ & $4,5 \mathrm{c}$ \\
\hline UFLA07-29 & $42,00 \mathrm{c}$ & $31,45 \mathrm{c}$ & $13,05 \mathrm{c}$ & $1,0377 \mathrm{~d}$ & $72,00 \mathrm{~d}$ & $17,06 \mathrm{a}$ & $12,23 \mathrm{c}$ & $21,85 \mathrm{~b}$ & $3,5 \mathrm{~b}$ \\
\hline UFLA07-31 & $56,80 \mathrm{~b}$ & $27,65 \mathrm{c}$ & $15,70 \mathrm{c}$ & $1,0333 \mathrm{~d}$ & $117,40 \mathrm{~d}$ & $15,41 \mathrm{a}$ & $18,61 \mathrm{~b}$ & $27,10 \mathrm{~b}$ & $1,5 \mathrm{a}$ \\
\hline UFLA07-37 & $18,20 \mathrm{~d}$ & $28,00 \mathrm{c}$ & $5,15 \mathrm{e}$ & $1,0615 \mathrm{~b}$ & $6,30 \mathrm{e}$ & $22,66 \mathrm{a}$ & $1,45 \mathrm{~d}$ & $13,45 \mathrm{c}$ & $4,0 \mathrm{~b}$ \\
\hline UFLA $07-40$ & $7,30 \mathrm{e}$ & $27,40 \mathrm{c}$ & $2,05 \mathrm{e}$ & $1,0690 \mathrm{~b}$ & $14,35 \mathrm{e}$ & $14,76 \mathrm{a}$ & $2,13 \mathrm{~d}$ & $4,30 \mathrm{c}$ & $2,5 \mathrm{a}$ \\
\hline UFLA07-41 & $7,20 \mathrm{e}$ & $40,30 \mathrm{a}$ & $2,90 \mathrm{e}$ & $1,0692 \mathrm{~b}$ & $86,45 \mathrm{~d}$ & $16,58 \mathrm{a}$ & $14,11 \mathrm{c}$ & $2,55 \mathrm{c}$ & $2,5 \mathrm{a}$ \\
\hline UFLA07-42 & $42,80 \mathrm{c}$ & $28,85 \mathrm{c}$ & $12,30 \mathrm{c}$ & $1,0601 \mathrm{~b}$ & $53,45 \mathrm{e}$ & $16,36 \mathrm{a}$ & $8,81 \mathrm{c}$ & $35,65 \mathrm{a}$ & $5,0 \mathrm{c}$ \\
\hline UFLA07-43 & $95,10 \mathrm{a}$ & $32,90 \mathrm{~b}$ & $31,60 \mathrm{a}$ & $1,0680 \mathrm{~b}$ & $115,40 \mathrm{~d}$ & $17,55 \mathrm{a}$ & $20,25 \mathrm{~b}$ & $42,70 \mathrm{a}$ & $4,0 \mathrm{~b}$ \\
\hline UFLA07-45 & $3,85 \mathrm{e}$ & $29,70 \mathrm{c}$ & $1,15 \mathrm{e}$ & $1,0993 \mathrm{a}$ & $3,20 \mathrm{e}$ & $17,85 \mathrm{a}$ & $0,57 \mathrm{~d}$ & $2,20 \mathrm{c}$ & $4,0 \mathrm{~b}$ \\
\hline UFLA07-46 & $22,00 \mathrm{~d}$ & $28,25 \mathrm{c}$ & $6,20 \mathrm{~d}$ & $1,0318 \mathrm{~d}$ & $19,70 \mathrm{e}$ & $15,54 \mathrm{a}$ & $3,04 \mathrm{~d}$ & $19,65 \mathrm{~b}$ & $4,5 \mathrm{c}$ \\
\hline UFLA07-48 & $16,90 \mathrm{~d}$ & $28,87 \mathrm{c}$ & $4,86 \mathrm{e}$ & $1,0487 \mathrm{c}$ & $24,14 \mathrm{e}$ & $15,01 \mathrm{a}$ & $2,81 \mathrm{~d}$ & $0,00 \mathrm{c}$ & $2,9 \mathrm{a}$ \\
\hline UFLA07-49 & $64,30 \mathrm{~b}$ & $32,70 \mathrm{~b}$ & $21,00 \mathrm{~b}$ & $1,0666 \mathrm{~b}$ & $84,00 \mathrm{~d}$ & $14,50 \mathrm{a}$ & $12,32 \mathrm{c}$ & $44,50 \mathrm{a}$ & $1,5 \mathrm{a}$ \\
\hline UFLA07-51 & $14,30 \mathrm{e}$ & $21,75 \mathrm{~d}$ & $3,10 \mathrm{e}$ & $1,0580 \mathrm{~b}$ & $10,25 \mathrm{e}$ & $16,88 \mathrm{a}$ & $1,72 \mathrm{~d}$ & $14,30 \mathrm{c}$ & $4,5 \mathrm{c}$ \\
\hline UFLA07-53 & $45,50 \mathrm{c}$ & $31,60 \mathrm{c}$ & $14,45 \mathrm{c}$ & $1,0665 \mathrm{~b}$ & $102,90 \mathrm{~d}$ & $16,39 \mathrm{a}$ & $16,83 \mathrm{~b}$ & $26,05 \mathrm{~b}$ & $1,5 \mathrm{a}$ \\
\hline Média & 29,51 & 31,50 & 8,90 & 1,0511 & 78,58 & 16,32 & 12,23 & 16,50 & 3,28 \\
\hline
\end{tabular}

${ }^{1}$ Testemunha (control treatment); ${ }^{2}$ Valores baixos são desejáveis (low values are desirable); *Médias com a mesma letra na coluna não diferem entre si, pelo teste de Scott-Knott ao nível de 5\% de probabilidade (means with the same letter in column are not different through the Scott-Knott test at $5 \%$ ). 
Tabela 3. Estimativas da herdabilidade no sentido amplo $\left(\mathrm{h}_{\mathrm{a}}^{2}\right)$, coeficiente de variação genético $\left(\mathrm{CV}_{\mathrm{g}}\right)$, coeficiente de variação ambiental, razão entre coeficiente de variação genético e ambiental $\left(\mathrm{CV}_{\mathrm{g}} / \mathrm{CV}_{\mathrm{e}}\right)$ em caracteres de clones de batata-doce (estimates of broad sense heritabilities $\left(\mathrm{h}_{\mathrm{a}}{ }^{2}\right)$, genetic coefficients of variation $\left(\mathrm{CV}_{\mathrm{g}}\right)$, environmental coefficients of variation $\left(\mathrm{CV}_{\mathrm{e}}\right)$, and ratio of genetic/envirornmental coefficients of variation $\left(\mathrm{CV}_{\mathrm{g}} / \mathrm{CV}_{\mathrm{e}}\right)$ for sweetpotato traits). Ijaci, Hortiagro, 2008.

\begin{tabular}{lcccccccc}
\hline Parâmetros & $\begin{array}{c}\text { Massa seca } \\
\text { das raízes } \\
(\%)\end{array}$ & $\begin{array}{c}\text { Produção } \\
\text { total de raízes } \\
\text { frescas }\end{array}$ & $\begin{array}{c}{ }^{\mathbf{o}} \text { Brix das } \\
\text { raízes }\end{array}$ & $\begin{array}{c}\text { Nota para } \\
\text { formato geral } \\
\text { de raízes }\end{array}$ & $\begin{array}{c}\text { Nota para } \\
\text { formato médio } \\
\text { de raízes }\end{array}$ & $\begin{array}{c}\text { Densidade de } \\
\text { raízes }\end{array}$ & $\begin{array}{c}\text { Produção de } \\
\text { massa fresca } \\
\text { da parte aérea }\end{array}$ & $\begin{array}{c}\text { Massa seca na } \\
\text { parte aérea } \\
\text { (\%) }\end{array}$ \\
\hline $\mathrm{h}_{\mathrm{a}}^{2}$ & 85,00 & 95,72 & 89,95 & 79,34 & 66,86 & 87,53 & 95,90 \\
$\mathrm{CV}_{\mathrm{g}}$ & 15,41 & 71,79 & 12,13 & 29,01 & 23,79 & 1,51 & 80,76 & 49,77 \\
$\mathrm{CV}_{\mathrm{e}}$ & 8,83 & 21,47 & 5,52 & 20,18 & 22,82 & 0,78 & 23,61 & 13,07 \\
$\mathrm{CV}_{\mathrm{g}} / \mathrm{CV}_{\mathrm{e}}$ & 1,75 & 3,34 & 2,20 & 1,44 & 1,04 & 1,94 & 3,42 & 0,73 \\
\hline
\end{tabular}

e in natura (Maeda \& Dip, 2000). E, como o teor de massa seca influencia diretamente no rendimento industrial, é importante verificar se na batata-doce esta relação também existe, já que densidade poderia ser utilizada como indicativo do rendimento etílico, sendo esta um caractere de fácil mensuração.

$\mathrm{Na}$ produção de massa fresca de parte aérea, a testemunha mais produtiva Brazlandia-Rosada produziu 101,80 t $\mathrm{ha}^{-1}$, ao passo que os clones mais produtivos ultrapassaram $200 \mathrm{t} \mathrm{ha}^{-1}$ e o melhor deles (UFLA07-15) chegou a 302,45 t $\mathrm{ha}^{-1}$ de massa fresca de parte aérea (Tabela 2). Estes dados são bem superiores aos relatados por Cardoso et al. (2005) e Queiroga et al. (2007), cujos valores máximos encontrados foram 14,10 e 14,58 tha $^{-1}$, respectivamente, realizando colheita aos 5 e 6 meses.

Não houve diferença estatística entre os clones para a porcentagem de massa seca da parte aérea que variou de 11,45 a 22,66\%. Valores muito baixos de massa seca podem limitar o consumo de forragem, devido à elevada ingestão de água no material. Outra consequência do baixo nível de massa seca, é que, para o material ser ensilado é necessário adicionar outros materiais para aumentar essa característica.

Para produção de massa seca na parte aérea, os clones mais produtivos foram UFLA07-08 (34,83 $\left.\mathrm{t} \mathrm{ha}^{-1}\right)$, UFLA07-15 (34,41 t ha-1), UFLA07-21 $\left(36,08 \mathrm{t} \mathrm{ha}^{-1}\right)$ e UFLA07-24 (36,10 t $\left.\mathrm{ha}^{-1}\right)$, ao passo que a melhor das testemunhas, Brazlândia-Rosada, produziu 20,01 t ha-1 (Tabela 2).

A produção de raízes comerciais apresentou grande amplitude de variação (0 a 51,35 t ha-1). Das testemunhas, a mais produtiva foi a Brazlândia-
-Branca, que alcançou 24,60 tha he raízes comerciais. Vários clones não diferiram estatisticamente das testemunhas (Tabela 2), enquanto que os clones mais produtivos apresentaram 35,65 (UFLA07-42); 42,70 (UFLA0743); 44,50 (UFLA07-49) e 51,35 t ha-1 (UFLA07-12). Estes resultados são bem mais altos que os encontrados por Azevedo et al. (2000) e Cardoso et al. (2005). Uma explicação para parte das diferenças de produtividade, tanto de produção de raízes comerciais como de outras características, é que normalmente outros autores colhem batata-doce 4 ou 5 meses após o plantio, ao passo que neste ensaio tal colheita foi realizada 7 meses pós plantio. Resende (2000) e Queiroga et al. (2007) também obtiveram aumentos na produtividade em experimentos com batata-doce colhidas mais tardiamente.

Para formato médio das raízes, apesar da diferença significativa $(p<0,05)$ entre os genótipos, em geral quando comparados com as testemunhas, os clones de batata-doce mostraram-se com um bom potencial para formato de raiz, com muitos clones não diferindo das testemunhas comerciais, exceto os clones, UFLA07-10, UFLA07-18, UFLA07-21, UFLA07-22, UFLA07-26, UFLA07-27 e UFLA07-42 que foram estatisticamente inferiores (Tabela 2).

O coeficiente de variação experimental $\left(\mathrm{CV}_{\mathrm{e}}\right)$ é o parâmetro que indica a magnitude da precisão experimental em experimentos. Neste aspecto, observou-se que os valores dos $\mathrm{CV}_{\mathrm{e}}$ (Tabela 3) estão dentro da faixa encontrada para a cultura da batata-doce. Cardoso et al. (2005) e Queiroga et al. (2007) encontraram CV acima de $40 \%$ avaliando dentre outros, caracteres de produtivi- dade de raízes e parte aérea de clones de batata-doce. As estimativas para o coeficiente de variação genética foram bem mais elevadas que para o coeficiente de variação ambiental nos caracteres avaliados, exceto para porcentagem da massa seca na parte aérea, demonstrando alta variabilidade entre os genótipos e uma situação bastante favorável para a seleção. Além disso, as estimativas de herdabilidade no sentido amplo foram superiores a 79\% para todos os caracteres analisados, com exceção da porcentagem da massa seca na parte aérea e nota para formato médio de raízes, e os valores da razão $b=\mathrm{CV}_{\mathrm{g}}$ ' $\mathrm{CV}_{\mathrm{e}}$, foram superiores a 1,0 , exceto para porcentagem da massa seca na parte aérea (Tabela 3), o que mais uma vez indica alta variabilidade e situação favorável para a seleção (Ferreira et al., 2003).

Com relação às correlações, para todos os pares de caracteres estudados os coeficientes de correlação genotípica e fenotípica, além de ser de mesmo sinal, foram semelhantes na magnitude e no nível de significância. Das estimativas que apresentaram correlações significativas, as correlações genotípicas foram ligeiramente superiores às fenotípicas como, produção total de raízes frescas x porcentagem de massa seca das raízes; porcentagem de massa seca na parte aérea x produção total de raízes frescas; nota para formato médio de raízes $\mathrm{x}$ nota para formato geral de raízes. E ambas, em todos os casos, foram superiores às correlações de ambiente (Tabela 4). Pode-se, portanto, concluir que há uma maior contribuição dos fatores genéticos que das ambientais nas estimativas das correlações fenotípicas entre os caracteres estudados. 
Tabela 4. Matriz de correlações fenotípicas $\left(\mathrm{r}_{\mathrm{F}}\right)$, genotípicas $\left(\mathrm{r}_{\mathrm{G}}\right)$ e ambientais $\left(\mathrm{r}_{\mathrm{A}}\right)$ entre caracteres de 39 clones de batata-doce (matrix of phenotipic $\left(\mathrm{r}_{\mathrm{F}}\right)$, genotypic $\left(\mathrm{r}_{\mathrm{G}}\right)$ and environmental $\left(\mathrm{r}_{\mathrm{A}}\right)$ correlations among traits of 39 sweet potato clones). Ijaci, Hortiagro, 2007.

\begin{tabular}{lcccccc}
\hline Caracteres & $\mathbf{r}$ & $\begin{array}{c}\text { Produção } \\
\text { total de raízes } \\
\text { frescas }\end{array}$ & $\begin{array}{c}\text { Densidade } \\
\text { de raízes }\end{array}$ & $\begin{array}{c}\text { Produção de } \\
\text { massa fresca } \\
\text { da parte aérea }\end{array}$ & $\begin{array}{c}\text { Massa seca na } \\
\text { parte aérea } \\
\mathbf{( \% )}\end{array}$ & $\begin{array}{c}\text { Nota para } \\
\text { formato médio de } \\
\text { raízes }\end{array}$ \\
\hline $\begin{array}{l}\text { Porcentagem de } \\
\text { Massa seca das }\end{array}$ & $\mathrm{F}$ & $-0,340^{+}$ & 0,291 & - & - & - \\
raízes & $\mathrm{G}$ & $-0,364^{+}$ & 0,285 & - & - & - \\
\hline Produção total de & $\mathrm{F}$ & $-0,199$ & $0,349^{+}$ & - & $-0,265^{*}$ & - \\
raízes frescas & $\mathrm{G}$ & - & - & 0,271 & $-0,344^{+}$ & - \\
\hline \multirow{2}{*}{$\begin{array}{l}\text { Nota para formato } \\
\text { geral de raízes }\end{array}$} & $\mathrm{F}$ & - & - & $-0,032^{+}$ & -155 & - \\
\hline
\end{tabular}

${ }^{* * *}$, Significativo a 1 e $5 \%$, pelo teste $\mathrm{t}$, respectivamente (significant at $1 \%$ and $5 \%$ through the $\mathrm{t}$ test, respectively); ${ }^{++},{ }^{+}$Significativo a $1 \mathrm{e}$ $5 \%$, respectivamente, pelo método de bootstrap com 10.000 simulações (significant at 1 and 5\% through the bootstrap method with 10,000 simulations).

Uma estimativa de correlação genotípica positiva e alta entre caracteres demonstra que na prática tem-se necessidade da avaliação apenas do caráter de mais fácil determinação, pois a seleção estará sendo realizada de forma indireta também para o outro caráter. Pode-se inferir com as altas estimativas que os genes que controlam um caráter podem ser os mesmos que controlam o outro (pleiotropia), ou esses, por sua vez, estão ligados. Informações como estas são de grande importância prática, reduzindo assim o tempo gasto com avaliações, como foi verificado com relação à estimativa de correlação genética entre nota para formato geral de raízes e nota para formato médio de raízes.

A produção total de raízes frescas apresentou estimativa de correlação significativa, porém com sinal negativo, com porcentagem da massa seca na parte aérea $\left(r_{\mathrm{G}}=-0,344^{+}\right.$e $\left.\mathrm{r}_{\mathrm{F}}=-0,265^{*}\right)$ e também com porcentagem de massa seca das raízes $\left(r_{G}=-0,364^{+}\right.$e $\left.r_{F}=-0,340^{+}\right)$. Apesar da estimativa de correlação entre produção total de raízes fresca e porcentagem de massa seca das raízes ter sido significativa, os valores foram baixos e com baixo coeficiente de determinação (Tabela 4).

Não se verificou correlação genética ou fenotípica entre a porcentagem de massa seca das raízes e densidade de raízes. Com isso, ao contrário do esperado, a densidade das raízes avaliadas em balança hidrostática não foi um bom indicativo para porcentagem de massa seca, não havendo aparentemente grande relação entre as duas características. Também não houve correlações genéticas ou fenotípicas significativas entre produção total de raízes frescas e produção de massa fresca da parte aérea.

Nas estimativas de correlações ambientais, mesmo quando significativas, os valores foram baixos, como nas correlações porcentagem de massa seca das raízes $\mathrm{x}$ densidade de raízes e produção total de raízes frescas $\mathrm{x}$ produção de massa fresca da parte aérea. Já a estimativa de correlação ambiental entre nota para formato geral de raízes e nota para formato médio de raízes foi alta e significativa (Tabela 4). Isso demonstra que esses últimos caracteres são afetados de maneira semelhante pelas mesmas condições do ambiente (Falconer, 1981).

A seleção e recomendação de genótipos de batata-doce baseada no conjunto de algumas dessas características estudadas (dependendo do segmento de mercado a ser explorado), possibilitará a plena utilização deste valioso recurso genético vegetal, e a sua adoção mais intensa na agricultura nacional. Para produção de etanol os principais caracteres determinados foram produção de raízes frescas e produção de massa seca nas raízes, destacando-se os clones UFLA07-49, UFLA07-12 e
UFLA07-43. Para alimentação animal os caracteres mais importantes são produção de massa seca nas raízes e produção de massa seca na parte aérea, onde os melhores resultados foram obtidos pelos clones, UFLA07-08, UFLA07-12, UFLA07-15, UFLA07-21, UFLA0724, UFLA07-43 e UFLA07-49. Já para alimentação humana o principal caráter, produção de raízes comerciais, teve como clones mais produtivos UFLA07-12, UFLA07-42; UFLA07-43 e UFLA07-49.

Dessa maneira, seus múltiplos usos podem contribuir mais intensamente para o fornecimento de energia (etanol biocombustível), sem prejuízo para a melhoria da alimentação humana (quantitativa e qualitativamente) e integração agricultura-pecuária (por meio do aproveitamento mais intenso de ramas e raízes na alimentação animal).

\section{AGRADECIMENTOS}

À Fundação de Amparo à Pesquisa do Estado de Minas Gerais (FAPEMIG), ao Conselho Nacional de Desenvolvimento Científico e Tecnológico ( $\mathrm{CNPq})$, Ministério da Ciência e Tecnologia (MCT), à Coordenação de Aperfeiçoamento de Pessoal de Nível Superior (CAPES), à Universidade Federal de Lavras (UFLA), à Fundação de Apoio ao Ensino, Pesquisa e Extensão (FAEPE) 
e à empresa HortiAgro Sementes Ltda, pela concessão de bolsa, recursos financeiros e/ou infraestrutura para realização do trabalho.

\section{REFERÊNCIAS}

AZEVEDO SM; FREITAS JA; MALUF WR; SILVEIRA MA. 2000. Desempenho de clones e métodos de plantio de batata-doce. Acta Scientiarum 22: 901-905.

BIOETANOL de cana-de-açúcar: energia para o desenvolvimento sustentável. Rio de Janeiro: BNDES, 2008. Disponível em: <http://www. bioetanoldecana.org/pt/download/bioetanol. pdf>. Acesso em: 22 abr. 2011.

CARDOSO AD; VIANA AES; RAMOS PAS; MATSUMOTO SN; AMARAL CLF; SEDIYAMA T; MORAIS OM. 2005. Avaliação de clones de batata-doce em Vitória da Conquista. Horticultura Brasileira 23: 911-914.

CARDOSO AD; VIANA AES; MATSUMOTO SN; BONFIM NETO H; KHOURI CR; MELO TL. 2007. Características físicas e sensoriais de clones de batata-doce. Ciência e Agrotecnologia 31: 1760-1765.

CASTRO LAS; EMYGDIO BM; ABRANTES VL; ROCHA NEM; 2008. Acessos de batatadoce do banco ativo de germoplasma da Embrapa Clima Temperado, com potencial de produção de biocombustível. Pelotas: Embrapa Clima Temperado, p. 23 (Embrapa Clima Temperado. Documentos, 258).

CAVALCANTE M; VANDERLEI FERREIRAP; LOPES PS; SILVA MJA; GOMES PEREIRA
R; 2008. Caracterização e divergência genética da batata-doce por meio de marcadores morfológicos. Revista Caatinga 21: 4: 89-95.

CENTRO INTERNACIONAL DE LA PAPA. 2001. Sweetpotato facts. Lima. Disponível em http://www.cipotato.org/market/Sweetpfacts/ swtpfact.htm.

EFRON B; TIBSHIRANI RJ. 1993. An introduction to the bootstrap. London: Chapman and Hall. 436p.

FALCONER D. 1981. Introdução a genética quantitativa. Viçosa: UFV.

FERREIRA MAJF; QUEIROZ MA; BRAZ LT; VENCOVSKY R. 2003. Correlações genotípicas, fenotípicas e de ambiente entre dez caracteres de melancia e suas implicações para o melhoramento genético. Horticultura Brasileira 21: 438-442.

GONÇALVES NETO AC; MALUF WR; GOMES LAA; GONÇALVES RJS; SILVA VF; LASMAR A; 2011. Aptidões de genótipos de batata-doce para consumo humano, produção de etanol e alimentação animal. Pesquisa agropecuária Brasileira 46:11:1513-1520.

IBGE. INSTITUTO BRASILEIRO DE GEOGRAFIA E ESTATÍSTICA. 2010. Sistema de informação. Disponível em: http:// ibge.gov.br/estados/temas.

MAEDA M; DIP TM; 2000. Curvas de porcentagem mássica de água versus peso específico em vegetais in natura - otimização de processos industriais pela seleção via teste da matéria-prima. Ciência Tecnologia de Alimentos 20: 3.

MIRANDA JEC; FRANÇA FH; CARRIJO OA; SOUZA AF; PEREIRA W; LOPES CA; DILVA JBC. 1995. A cultura da batata-doce. Brasília: Embrapa/CNPH. 94p.
NEIVA IP; ANDRADE JÚNIOR VC; VIANA DJS; FIGUEIREDO JA; MENDONÇA FILHO CV; PARRELLA RAC; SANTOS JB. 2011. Caracterização morfológica de acessos de batata-doce do banco de germoplasma da UFVJM, Diamantina. Horticultura Brasileira 29: 537-541.

OLIVEIRAAP; MOURA MF; NOGUEIRA DH; CHAGAS NG; BRAZ MSS; OLIVEIRA MRT; BARBOSA JA. 2006. Produção de raízes de batata-doce em função do uso de doses de $\mathrm{N}$ aplicadas no solo e via foliar. Horticultura Brasileira 24: 279-282.

OLIVEIRA ACB; SEDIYAMA MAN; SEDIYAMA T; CRUZ CD. 2000. Avaliação da divergência genética em batata-doce por procedimentos multivariados. Acta Scientiarum 22: 895-900.

QUEIROGA RCF; SANTOS MA; MENEZES MA; VIEIRA CPG; SILVA MC. 2007. Fisiologia e produção de cultivares de batata-doce em função da época de colheita. Horticultura Brasileira 25: 371-374.

RESENDE GM. 2000. Características produtivas de cultivares de batata-doce em duas épocas de colheita, em Porteirinha, MG. Horticultura Brasileira 18: 68-71.

SAS INSTITUTE. 2001. SAS/STAT software: changes and enhancements. Release 8. Cary.

SILVEIRA MA; ALVIM TC; DIAS LD; ANDRÉ CMG; TAVARES IB; SANTANA WR; SOUZA FR. 2007. A cultura da batata-doce como fonte de matéria-prima para produção de etanol. Palmas: UFT. 45 p. (Boletim Técnico).

SILVEIRA MA. 2008. Batata-doce: uma nova alternativa para a produção de etanol. In: INSTITUTO EUVALDO LODI. Álcool combustível. Brasília: Núcleo Central. 1: 109-122. 